Article

\title{
A Multi-Channel Opto-Electronic Sensor to Accurately Monitor Heart Rate against Motion Artefact during Exercise
}

\author{
Abdullah Alzahrani ${ }^{1, \dagger}$, Sijung Hu ${ }^{1, \dagger}, *$, Vicente Azorin-Peris ${ }^{1, \dagger}$, Laura Barrett ${ }^{2}$, Dale Esliger ${ }^{2}$, \\ Matthew Hayes ${ }^{3}$, Shafique Akbare ${ }^{4}$, Jérôme Achart ${ }^{4}$ and Sylvain Kuoch ${ }^{4}$
}

1 Wolfson School of Mechanical, Manufacturing and Electrical Engineering,

Loughborough University, Ashby Road, Loughborough, Leicestershire LE11 3TU, UK;

E-Mails: A.Alzahrani@lboro.ac.uk (A.A.); V.Azorin-Peris@lboro.ac.uk (V.A.-P.)

2 National Centre for Sport and Exercise Medicine, School of Sport, Exercise and Health Sciences, Loughborough University, Ashby Road, Loughborough, Leicestershire LE11 3TU, UK;

E-Mails: L.A.Barrett@lboro.ac.uk (L.B.); D.Esliger@1boro.ac.uk (D.E.)

3 Cambridge Consultants Limited, Science Park, Milton Road, Cambridge CB4 0DW, UK;

E-Mail: matthew.hayes@cambridgeconsultants.com

4 Université Paris-Sud 11, Polytech'Paris-Sud-Service des stages, Bât 620, Orsaycedex 91405, France; E-Mails: shafique.akbare@gmail.com (S.A.); achart.j@gmail.com (J.A.); sylvain.kuoch@gmail.com (S.K.)

$\dagger$ These authors contributed equally to this work.

* Author to whom correspondence should be addressed; E-Mail: S.Hu@lboro.ac.uk; Tel.: +44-1509-227-059.

Academic Editors: Steffen Leonhardt and Daniel Teichmann

Received: 30 July 2015 / Accepted: 29 September 2015 / Published: 12 October 2015

\begin{abstract}
This study presents the use of a multi-channel opto-electronic sensor (OEPS) to effectively monitor critical physiological parameters whilst preventing motion artefact as increasingly demanded by personal healthcare. The aim of this work was to study how to capture the heart rate (HR) efficiently through a well-constructed OEPS and a 3-axis accelerometer with wireless communication. A protocol was designed to incorporate sitting, standing, walking, running and cycling. The datasets collected from these activities were processed to elaborate sport physiological effects. t-test, Bland-Altman Agreement (BAA), and correlation to evaluate the performance of the OEPS were used against Polar and Mio-Alpha HR monitors. No differences in the HR were found between OEPS, and either Polar or Mio-Alpha (both $p>0.05$ ); a strong correlation was found between
\end{abstract}


Polar and OEPS (r: 0.96, $p<0.001$ ); the bias of BAA $0.85 \mathrm{bpm}$, the standard deviation (SD) $9.20 \mathrm{bpm}$, and the limits of agreement (LOA) from $-17.18 \mathrm{bpm}$ to $+18.88 \mathrm{bpm}$. For the Mio-Alpha and OEPS, a strong correlation was found (r: 0.96, $p<0.001$ ); the bias of BAA $1.63 \mathrm{bpm}$, SD $8.62 \mathrm{bpm}$, LOA from $-15.27 \mathrm{bpm}$ to $+18.58 \mathrm{bpm}$. These results demonstrate the OEPS to be capable of carrying out real time and remote monitoring of heart rate.

Keywords: multi-channel opto-electronics; accelerometer; motion artefact; sport physiological monitoring; heart rate; pulse wave; vital signs; wearables

\section{Introduction}

People suffering from cardiovascular disease (CVD) need to observe their health conditions continuously in order to prevent any further deterioration, to determine current health status [1] and to maintain patients' quality of life [2]. In today's growing and ageing population, cardiovascular disease, stroke and diabetes are the main causes of disability and death [3]. Home and Tele-monitoring can be a cost-effective solution in forthcoming healthcare, and could help elder and illness people maintaining their quality life [4]. Tele-monitoring together with home-delivered care is an inevitable trend and becoming a valuable means to reduce the costs of treatment and to increase service quality in our routine healthcare world [5].

Monitoring of physiological parameters such as heart rate (HR) is also crucially important in many sports and exercise applications in order to monitor training and to ensure athletes are training at the right intensity. In addition, monitoring $\mathrm{HR}$ and heart rate variability (HRV) could potentially play a vital role in the prevention and detection of overtraining [6]. HR monitoring is also used as a means of objectively monitoring physical activity levels and as a means of estimating energy expenditure [7]. Photoplethysmography (PPG) is a non-invasive optical technique used to measure blood volume change in micro-vascular beds of tissue [8]. Photoplethysmography can be used to monitor health vital signs, such as HR, HRV, respiration rate (RR), blood pressure (BP) and oxygen saturation $\left(\mathrm{SpO}_{2} \%\right)$ [9]. The principle of detection can be described with Lambert Beer's law to predict the intensity of red and IR light transmitted through and reflected/refracted from a pulsatile area of the body, such as a finger, forehead, in-ear or wrist onto a photo detector $[8,10]$. Figure 1 shows the absorption of pulsatile tissues as $\mathrm{AC}$ and non-pulsatile as steady DC absorption.

Wearable health monitors has recently become a popular and interesting topic among researchers and commercial companies. Many wearable devices such as FlexPock device [2], MAIN Shirt (MAIN: short for magnetic induction) [4], AMON (Advanced Medical Monitor) physiological monitor [11], Nonin Wrist Ox3100 (Nonin Medical, Inc., Plymouth, MN, USA) [12] SenseWear (Pittsburgh, PA, USA) [13], Smart Shirt (Sensatex Inc., Bethesda, MD, USA) [14], LifeShirt (Vivometrics Inc., Ventura, CA, USA) [15], and Smart Vest (Electromed, Simi Valley, CA, USA) [16] have been developed with various structures and electronic systems. Many of the devices are yet to provide a high performance incorporated with, and real time monitoring functionalities during an extensive movement $>8 \mathrm{~km} / \mathrm{h}$. For example, AMON wrist-wear is utilized for high risk patients [17] in bed or during normal walking, 
such as with elderly people, but not for jogging. Moreover, the data generated from Nonin Wrist Ox3100 [12] band is processed offline, as the data are stored locally in non-volatile memory and are extracted and analyzed at a later stage. This could lead to delays in diagnosis and is considered a limitation. Despite their wealth of physiological information, reliable PPG signals can be subject to heavy interference originating from different sources, including modification of the optical properties of the internal tissues, poor blood perfusion, and external sources such as electromagnetic and electronic noise, physical movement, misalignment between skin surface and optical sensor path and ambient light. Some artefact noises could be dealt with pre-processing though utilization of digital filters and ambient light subtraction approach as one of major challenges in PPG signal processing is motion induced error. In this case, motion artefact can influence the measurement signal and lead to a disrupted readout [8]. Lee et al. [18] indicated that the greatest and worst cause of artefact noise that contaminates the PPG signals is motion artefact (MA) produced from physical activity and body movement. Thus, various signal processing techniques have been proposed to remove MA and recover the PPG signals.

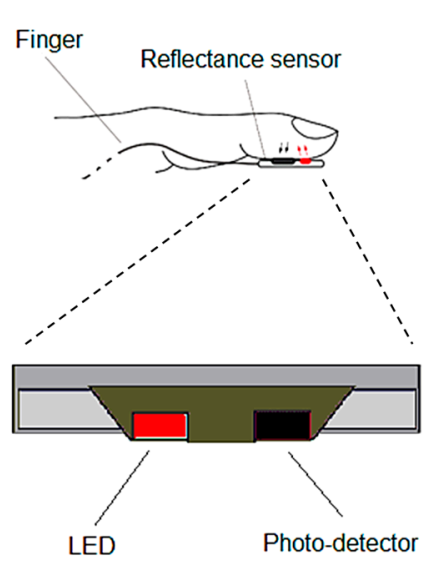

(a)

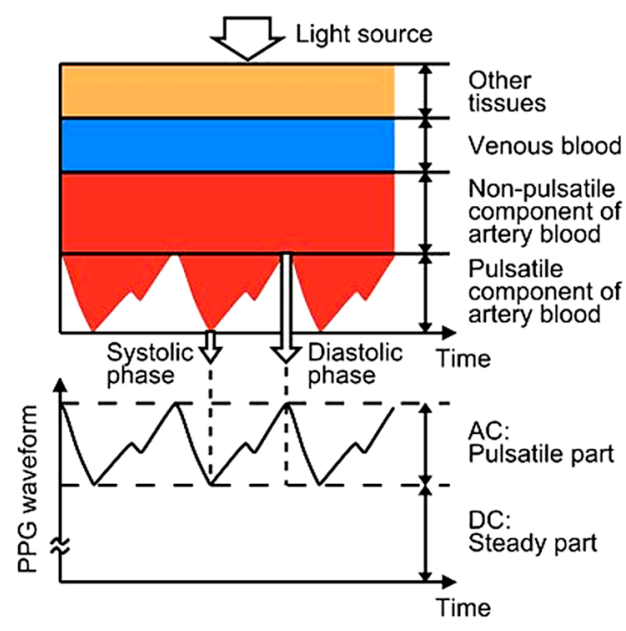

(b)

Figure 1. Illustrated diagram of the reflectance sensor (a) and the light absorbance of tissue components (b) (revisited and modified from [19]).

When noise is out of the range of physiological signals, average filtrating [20] is a filtration technique usually used to remove the noise. However this technique sometime cannot work effectively when the noise is near or shares the same frequency band as the signal components of interest as this technique can suppress the desired signals. Another approach is independent component analysis (ICA) as recommended by Yoo et al. [20] to use a block interleaving and basic ICA algorithm. Natarajan et al. [21] proposed an application of ICA in the frequency-domain as it is assumed that the subcomponents are non-Gaussian signals and are statistically independent from each other, thus the ICA does not capture PPG signals contaminated by MA. The periodogram method [22] has been used to estimate the HR with some drawbacks of inconsistent spectrum, high variances as well as serious leakage effects [23].

The adaptive noise cancelation (ANC) was also proposed to remove the motion artefact as Ram et al. [24] and Yousefi et al. [25] suggested using ANC to eliminate MA. To implement ANC, the 
system needs a higher computational memory, and the performance of ANC is sensitive to a reference signal. Among these techniques, the acceleration data (AD) could be considered at present as one of operative techniques to reduce MA. Fukushima et al. [26] suggested using a spectrum subtraction method to cancel the spectrum of acceleration data from the desired PPG signal. The AD with Kalman filtering (KF) as indicated by Lee et al. [27] could also reconstruct the signal from MA. Further techniques to eliminate or reduce MA include empirical mode decomposition (EMD) [28], electronic processing methodology [29], evaluation wavelet [30], and time-frequency analysis [31]. Despite the importance of signal processing, these methods cannot be used in many scenarios due to their implicit assumption that the corruption manifests itself as an additional signal component unrelated to physiological status either in time, frequency or statistical domains [32]. Most of these techniques were proposed and carefully set up for specific scenarios when users performed designated instructions with specific motions, e.g., keyboard typing, finger movements [23,24,33] and walking [25]. Thus these techniques might perform well when users make intensive physical movements such as those made during exercise. In addition, some studies were conducted by Poh et al. [34] and Lopez et al. [35] aimed to reduce MA and estimate HR in the presence of movement. Both studies focused on slow running at speed $<8 \mathrm{~km} / \mathrm{h}$ while Lopez et al. [35] utilized the earlobe as the site to detect PPG signals, where less MA is exhibited. Hence, the above studies have merely concentrated on physical activities under constraints of slow movement.

A multi-channel opto-electronic patch sensor (OEPS) [36] was employed in the study. OEPS is the outcome of opto-physiological interaction work to specifically study the effluence of tissue optical properties on blood driven dynamic changes named as opto-physiological modelling (OPM) [37]. An optimal optical sensing layout together a plurality of light sources with one photodetector configured to capture radiation after it has passed through the tissue is one of key factors to effectively capture these dynamic changes and the OEPS [38] was led by the OPM driven photoplethysmographic measurement studies $[39,40]$. Hence the OPES comprises of the following aspects:

1. The tissue optics properties to determine an optimal optical sensing position;

2. An optimal optical layout for the OEPS operable to monitor the tissue optic properties of the tissue type; and

3. Optical design involving in the options of a wavelength, intensity and an optical path length for the LED illumination sources.

Meanwhile, an analogue three-axis MEMS accelerometer (3MA, ADXL337, Analog Devices Inc., Norwood, MA, USA) was employed to detect acceleration and movement in all three axes with respect to gravitational acceleration and to provide a reliable movement reference. The 3MA provides higher accuracy in the characterization of physical movement with its three signal outputs for accelerations along spatial vectors $\mathrm{X}, \mathrm{Y}$ and $\mathrm{Z}$. The value of $3 \mathrm{MA}$ stems from the fact that capacitance changes inside the accelerometer reflect forces on all three axes in respect of gravitational acceleration. The total acceleration vector is produced as a combination of vectors $\mathrm{X}, \mathrm{Y}$ and $\mathrm{Z}$. When the user's body moves, the accelerometer produces signals proportional to the magnitude of the movement, and the resultant total acceleration vector $\mathrm{R}$ represents the instantaneous direction and magnitude of motion in $3 \mathrm{D}$ space. 
The purpose of this study was to assess HR by means of OEPS with sixteen healthy subjects across a range of physical activities, e.g., resting, cycling, jogging and running. A customized OEPS was attached to different measuring sites of the subject, e.g., palm, finger, forehead and back where the wearers perform intensive physical activities such as running up to $14 \mathrm{~km} / \mathrm{h}$ as well as cycling against various weight loads up to $2.5 \mathrm{~kg}$.

\section{Method}

\subsection{Experimental Setup of Opto-Electronic Patch Sensor (OEPS)}

The OEPS was constructed as a reflection opto-electronic sensor along with the outcome of opto-physiological modeling together with a specific optoelectronic system to reduce the impact of motion disturbance. The optimal optical design for the OPES is shown in Figure 2.

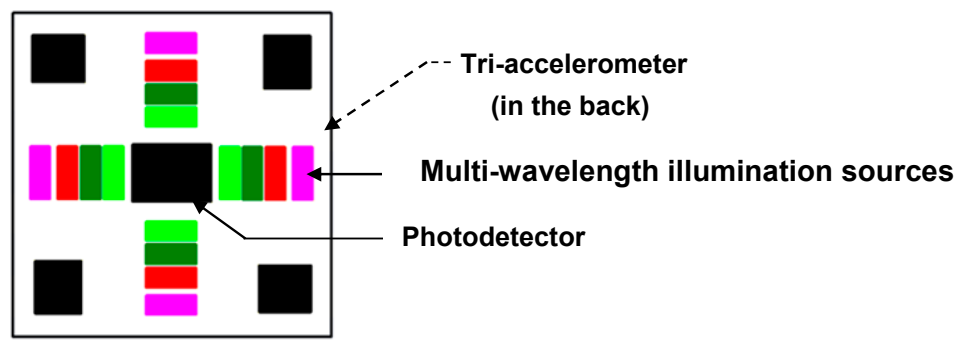

Figure 2. Schematic diagram of Opto-Electronic Patch Sensor (OEPS) with accelerometer.

The optimum separation of each of a plurality of light sources from a photodetector, based on modelled optical path lengths for light travelling from each light source, through the body tissue type to be monitored, to the photodetector; and locating light sources of different wavelengths at different distances from the photodetector. A reasonable distance between illumination sources and photo-sensor is required in order to avoid a saturation signal from a closer source and a reduced amplitude signal from a far light source [2]. A non-invasive OEPS is ideally configured for use with a body tissue type to be monitored so as to allow optical data, relating to the physiological parameters being studied, to be reliably and accurately obtained.

High gain, low noise and ultra-low power consumption in the OEPS electronic system was determined for continuous and real time monitoring. In Figure 3, the OEPS system shows a wearable health monitoring system wirelessly connected via a Bluetooth module to a Bluetooth-enabled cell phone/PC to perform further signal processing and display the outcomes of measurement.

Figure 3 illustrates the flow of OEPS electronics system. The output from the OEPS is passed through the analog front end (AFE). In the end, normal PPG signals are usually sent through a Bluetooth module. In the presence of movement, the module of motion artefact reduction (MAR) takes an execution action against a motion as the system structure takes the corrupted signals gained from the OEPS as a primary input and the motion signals from the 3-axis Micro-Electro-Mechanical Systems accelerometer (3MA) as a reference input.

To reach such an accurate level, the vector $\mathrm{R}$ of the accelerometer motions is mapped to the contribution of motion artefact. Actually, the accelerometer is most sensitive when the sensing axis is close to $0^{\circ}$ and less sensitive when the sensing axis is near $90^{\circ}$. 


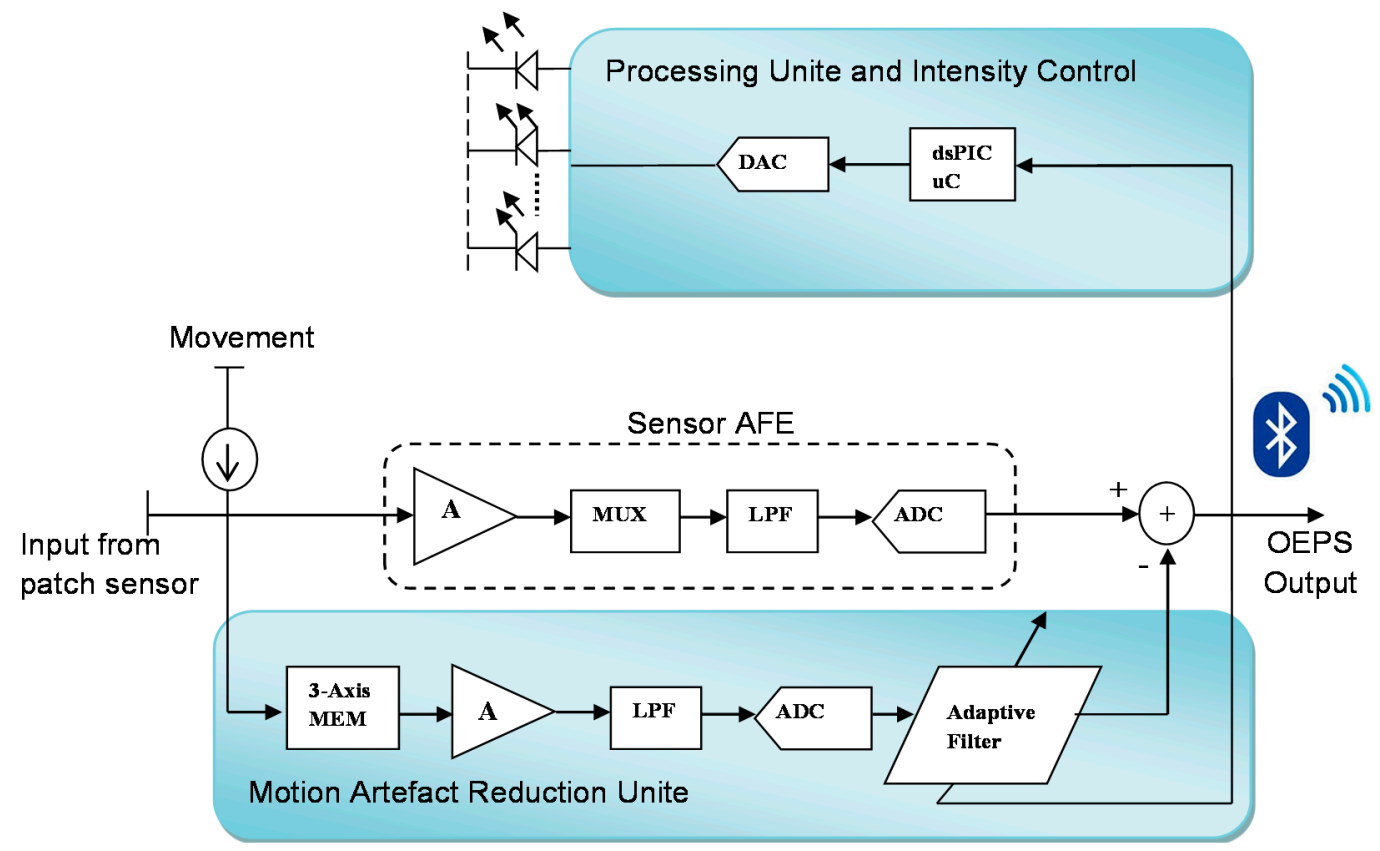

Figure 3. Electronic system structure of OEPS for continuous physiological monitoring.

Hence, the sensitivity could be preserved and compensated when combining $x$ and $y$ vectors as shown in Figure 4.

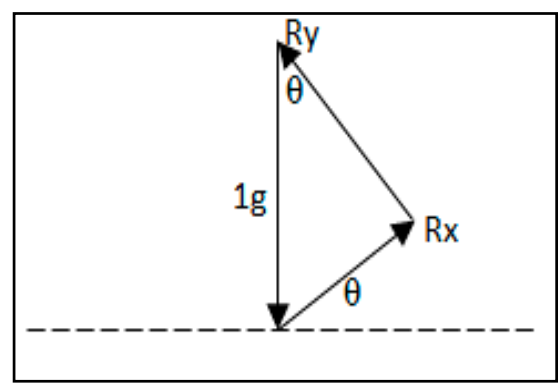

Figure 4. The combination of $x$ and $y$ vectors in two axis acceleration.

The combination of the vectors is equal to the output values of acceleration which are the projection of the force gravity as expressed on Equation (1).

$$
R=\sqrt{R x^{2}+R y^{2}}
$$

In this study, 3MA accelerometer was used to generate an accurate calibration of acceleration since the three vectors $x, y$ and $z$ were presented. The total acceleration vector is shown in Equation (2) and the combination vectors of $\mathrm{x}, \mathrm{y}$ and $\mathrm{z}$ is shown in Figure 5.

$$
R=\sqrt{R x^{2}+R y^{2}+R z^{2}}
$$

Thus, an effective cancellation method was introduced to reduce motion artefacts on corrupted PPG signals using the reference signals from the 3MA, followed by an adaptive filter to facilitate the optimum adaptive motion artefact cancellation technique as seen in Figure 3. To realize the adaptive noise cancellation, the OEPS system uses two inputs, e.g., the corrupted PPG signal as a primary input, and the combined motion signal of the accelerometer as a reference input. The motion signal is 
deducted from the primary input by the means of a close loop least mean square LMS adaptive filter to adjust its variable coefficients until the error is minimized to yield a recovered output signal since there is a difference between the output from the filter and the signal as desired. The adaptive filter for reducing motion artefacts was set to a filter order of 32 and a step size of $\mu: 0.1$.

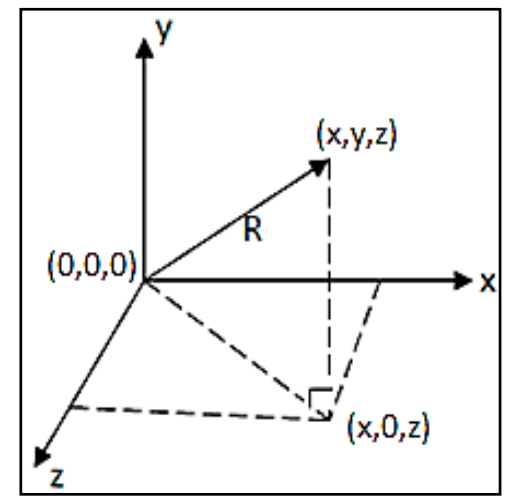

Figure 5. The combination of $x, y$ and $z$ vectors in three axis acceleration.

The OEPS consists of (1) an optical side to catch the backlight containing a serial of pulsatile signals; and (2) the electronic side with an analogue front-end to drive multi-wavelength LEDs and to pre-amplify the photodiode from (1), and the embedded digital for signal processing procedure and a communication module to wirelessly transmit the physiological outcomes to a computer and smartphone. The OEPS operates in reflectance mode (i.e., multi-wavelength illumination sources and the detector are on the same side) to make it suitable for attachment or adherence onto different locations of a human body, i.e., forehead, palm, earlobe and wrist, as presented in Figure 6. However, the amplitude of pulsatile signal in a reflectance mode is smaller than a conventional transmission mode; thus, a trans-impedance is required in order to convert photocurrent intensity into a proportional suitable voltage to enhance the signals generated from the photodiode [5].
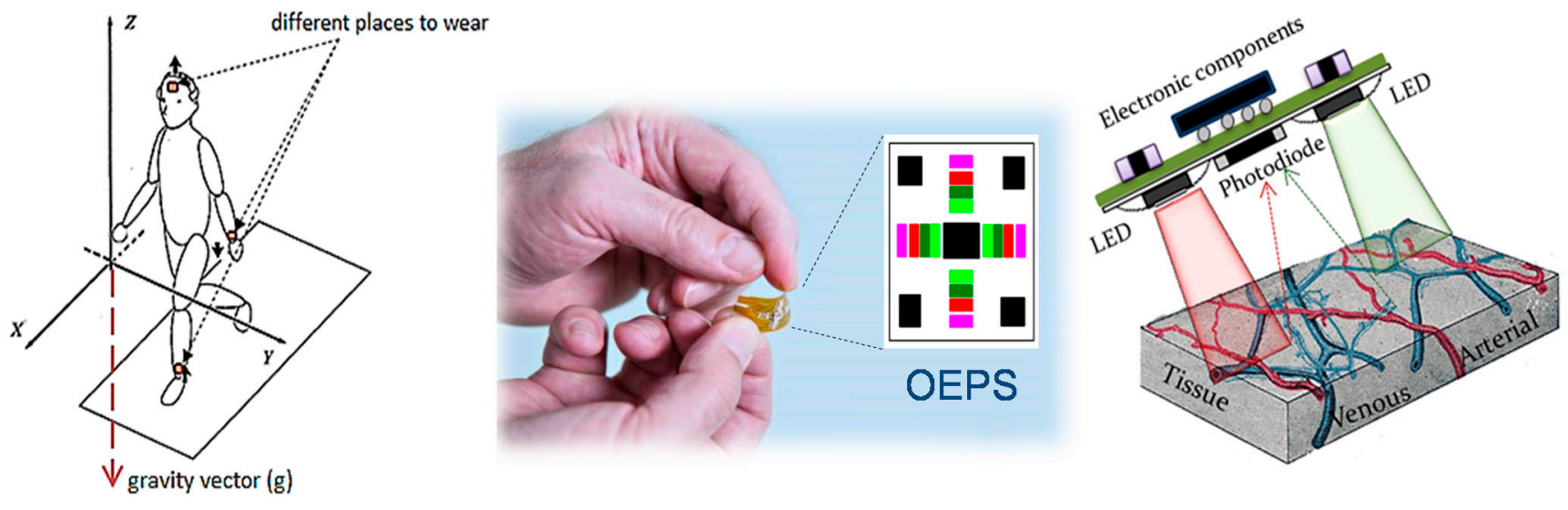

Figure 6. Flexible OEPS for continuous physiological monitoring and its viable locations.

Multi-wavelength illumination sources, i.e., LEDs of green $525 \mathrm{~nm}$, red $650 \mathrm{~nm}$ and IR $870 \mathrm{~nm}$ (JMSienna Co., Ltd., Palo Alto, CA, USA) were used for the OEPS including, a low-profile PiN photodiode (BPW34SR18R, Osram, GmbH). The analogue front-end consists of a pre-amplifier, a 
multiplexer and a low pass filter to performer-processing of the analogue signals gained from the OEPS to be prepared for analogue-to-digital conversion (ADC). A3MA (ADXL337, Analog Devices Co., Palo Alto, MA, USA) was employed to detect any motion from the body and the signal generated from the 3MA was used as a reference against MA. A dsPIC Microcontroller (dsPIC33FJ64GS610, Microchip Co., Chandler, AZ, USA) was hired to implement digital signal processing (DSP) and communication via a Universal Asynchronous Receiver/Transmitter (UART) protocol.

\subsection{Multiple Wavelength Illumination Source}

Since there is a variation in depth of penetration and absorption over the range of wavelengths [41], the intensity of the light penetrating through the skin and emerging from the tissue can be represented as the banana shape effect. Figure 7 shows the light path distribution of typical PPG model. $I_{i}$ is the light incident on the skin surface; $I_{0}$ is the intensity of light penetrating into the skin, $I_{r}$ is the reflected light intensity and $I$ is the intensity of light emerging from the tissue through the banana effect. Referring to the principle of Lambert-Beer's law, the light intensity passing through different layers of skin is decayed exponentially.

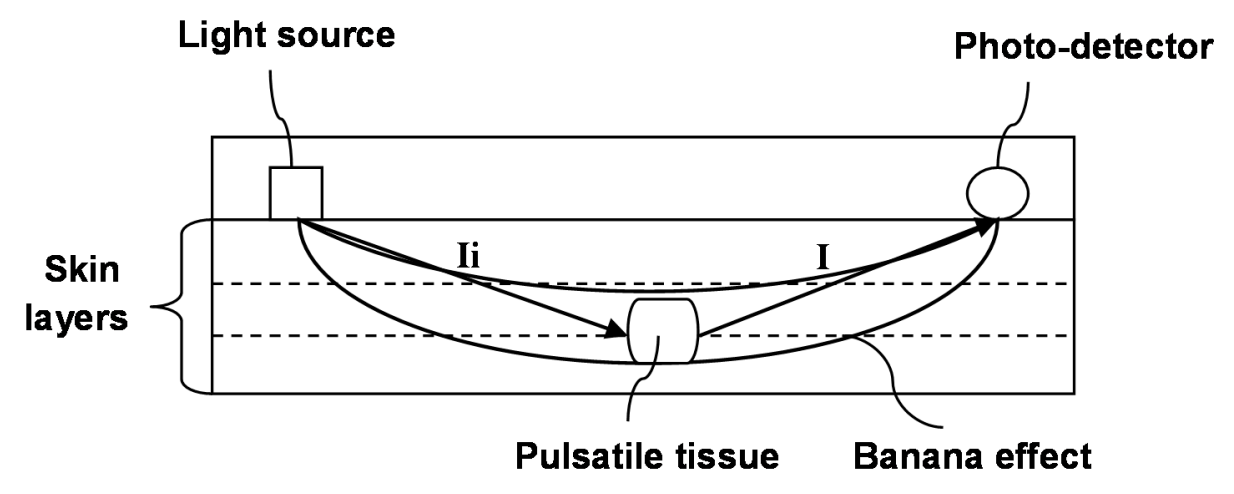

Figure 7. Banana shape effect on reflection mode OEPS.

The light back to the photo-detector can be separated into a non-dynamic (DC) component that results from non-pulsatile tissues and a dynamic (AC) component due to the presence of pulsatile blood volume changes. Thus, the model can be expressed as:

$$
I=I i \times e^{-\lambda t}=I i \times e^{-\left(\mu_{e f f} \times r\right) t}
$$

where $\lambda$ is the wavelength of a particular light, $I$ is the reflected light intensity after penetration, $I i$ is light incident, $\mu_{\text {eff }}$ is the absorption coefficient of both dynamic and static components, and $r$ is the light path length. Equation (3) can also be expressed as following formula

$$
I=I i \times e^{-\left(\mu_{d y} \times d(t)+\mu_{s t} \times m\right)}
$$

where $\mu_{d y}$ is the absorption coefficient of the dynamic and $\mu_{s t}$ is the static coefficient, the light path length through the dynamic and static components are $d(t)$ and $m$, respectively. The target is to enhance the pulsatile dynamic variation (PDV) upon different wavelengths and through multiple layers of the skin. The accuracy of PDV depends upon the quality of the AC component; however, the gain of PDV over the tissues (finger, ear-lobe) never exceeds 5\% of the incident light intensity [40]; even using super bright LEDs, the PDV light intensity remains on low values or could saturate the 
photo-detector (PD) due to the increase of reflected light $I_{r}$. Therefore, the $P D V$ should be maximized and this could be achieved by maximizing the light that travels or penetrates through the tissue $I$ and minimizing the reflection component of the static signal $I r$. The light intensities at the PD can be expressed as:

$$
I t=(I r+I o) \times e^{-\left(\mu_{d y} \times d(t)+\mu_{s t} \times m\right)}
$$

Expanding Equation 5 and splitting the dynamic and static term results in Equation (6).

$$
I t=(I r+I o) \times e^{-\mu_{s t} \times m\left(1+\mu_{d y} \times d(t)\right)}
$$

By applying a band pass filter with the cut-off frequency $0.18 \mathrm{~Hz}<f<10 \mathrm{~Hz}$ as in the design of $O E P S$, as a result any constant value and the $I_{r}$ can be eliminated. By normalizing the dynamic component with respect to the static component, Equation (7) can be used as the dynamic component becomes independent of the light source intensity.

$$
\frac{A C}{D C}=\frac{I o \times \mathrm{e}^{-\left(\mu_{s t} \times m\right) \times \mu_{d y} \times d(t)}}{I o \times \mathrm{e}^{-\left(\mu_{s t} \times m\right)}}=\mu_{d y} \times d(t)
$$

To obtain the optimum value of PDV, the absorption light is tested at the lab with multiple wavelengths and various distances between the peak of incidence light and the active area of the -detector. According to Lee [42] the green light PPG was found to have relative robustness against motion artefacts as compared with red and blue light PPG. Hence, the green wavelength is presented in this study as being more robust than the red and IR wavelength with the presence of movement.

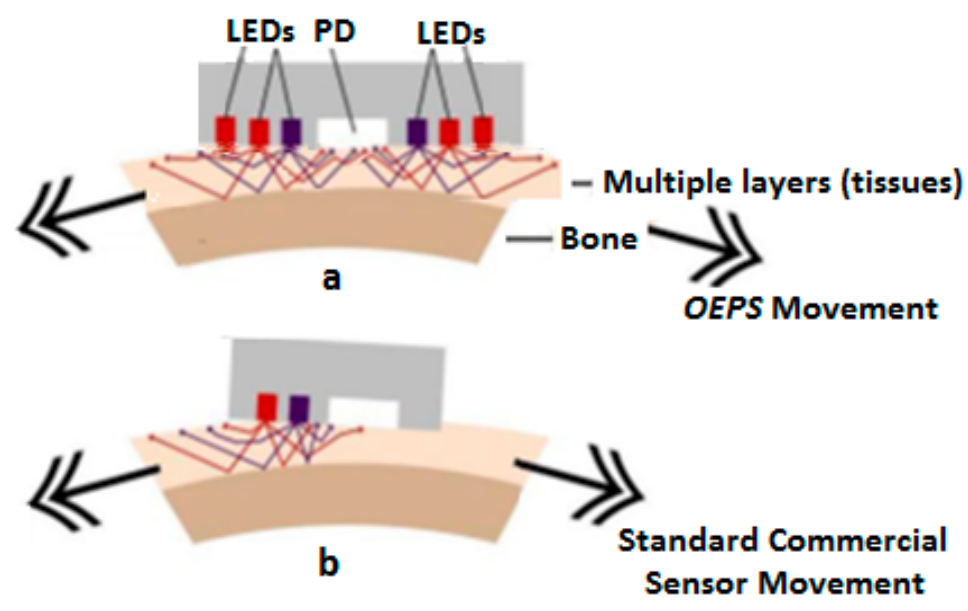

Figure 8. Sensor displacement altering backscattered light.

A multiple controlled LED modulator was created to stabilize the output of illumination of the OEPS patch sensor and to enhance the back-scattered light signals that are detected as seen in Figure 6. When the user moves in a random way the PVD can still be captured. Moreover, the OEPS can handle displacement and misalignment as indicated in Figure 8a. OEPS typically captures back-scattered light with movement because the OEPS can capture from other mirrored wavelength elements when one of the elements is being misaligned with the vessels. In contrast, Figure $8 \mathrm{~b}$ shows a standard commercial probe movement inducing and causing changes in the sensor position, changing the detected backscattered light. Additionally, the $3 \mathrm{MA}$ present in the system was used as a reference to reduce motion artefact [38]. 


\subsection{Physiological Monitoring Protocol}

Sixteen healthy active male participants (aged $24 \pm 3$ years, mean \pm standard deviation) volunteered to take part in the present investigation. The protocol was approved by the Loughborough University Ethical Advisory Committee and implemented in the sport physiological measurement laboratory in School of Sport, Exercise and Health Sciences, Loughborough University. Prior to any tests all participants had the procedures and possible risks associated with involvement in the study explained to them and their written consent to participant in the study was obtained. The measurements were taken while the participants were seated, while they walked and ran on a treadmill and cycled on a cycle ergometer. Gender (Male), Height $(179.8 \pm 4.1 \mathrm{~cm})$, body mass $(74.9 \pm 7.9 \mathrm{~kg})$,body mass index $\left(23.1 \pm 2.0 \mathrm{~kg} / \mathrm{m}^{2}\right)$ and blood pressure (systolic $130 \pm 10 \mathrm{mmHg}$, diastolic $67 \pm 9 \mathrm{mmHg}$ ) for individual participants as well as the room temperature $\left(24.4 \pm 0.5{ }^{\circ} \mathrm{C}\right)$ and humidity $(33 \% \pm 2 \%)$, were also measured during the experimental procedure.

HR was simultaneously monitored with a Polar Bluetooth ${ }^{\circledR}$ Smart chest strap ECG device, an accessory to Polar's Bluetooth ${ }^{\circledR}$ Smart wGT3X-BT activity monitor (Acti-Graph, LLC, Pensacola, FL, USA) as well as Mio-Alpha (Physical Enterprises Inc., Vancouver, BC, Canada) wrist watch HR monitor as the references. The back of OEPS sensor attached with 3MA was placed on the palm of the participant's left hand while the Mio-Alpha was worn on the left hand wrist. Both the OEPS and the commercial devices (Mio-Alpha and Polar) captured data simultaneously to facilitate the processing signals of and reference indications respectively. The participants were asked to rest in a seated and upright position for $180 \mathrm{~s}$, and the HR was then recorded over a $60 \mathrm{~s}$ period. Once the session of resting was completed to obtain HR, the participant started the exercise protocol on the treadmill (Technogym Excite Med 700, Gambettola, Italy).

The protocol consisted of (a) resting on the chair with a period of $180 \mathrm{~s}$; (b) walking and running on treadmill with an execution period of $540 \mathrm{~s}$; and (c) cycling exercise with loads implemented in period of $240 \mathrm{~s}$. The protocol for walking and running on treadmill composed of $1 \mathrm{st}$ session of walking exercise at a speed of $4 \mathrm{~km} / \mathrm{h}$ within the period of $180 \mathrm{~s}$; 2nd session of running exercise at a speed of $7 \mathrm{~km} / \mathrm{h}$ within the period of $180 \mathrm{~s}$; and 3rd session of faster running exercise at a speed of $8.5 \mathrm{~km} / \mathrm{h}$ within the period of $180 \mathrm{~s}$. The HR was detected by the OPES while the participant moved his left arm freely in each sessions including the resting and the walking and running. The participant was then asked to complete the cycling protocol consisting of four sessions (60 s each) cycling at the same speed of at $60 \mathrm{rpm}$ on a Gym cycle (Monark Ergomedic 874E, Vansbro, Sweden) with four mass loads, for instance. 1st session with $1.0 \mathrm{~kg}$, 2nd session with $1.5 \mathrm{~kg}$, 3rd session with $2.0 \mathrm{~kg}$, and 4th session with $2.5 \mathrm{~kg}$. The HR was recorded in each session. Figure 9 represents the entire exercises protocol. 


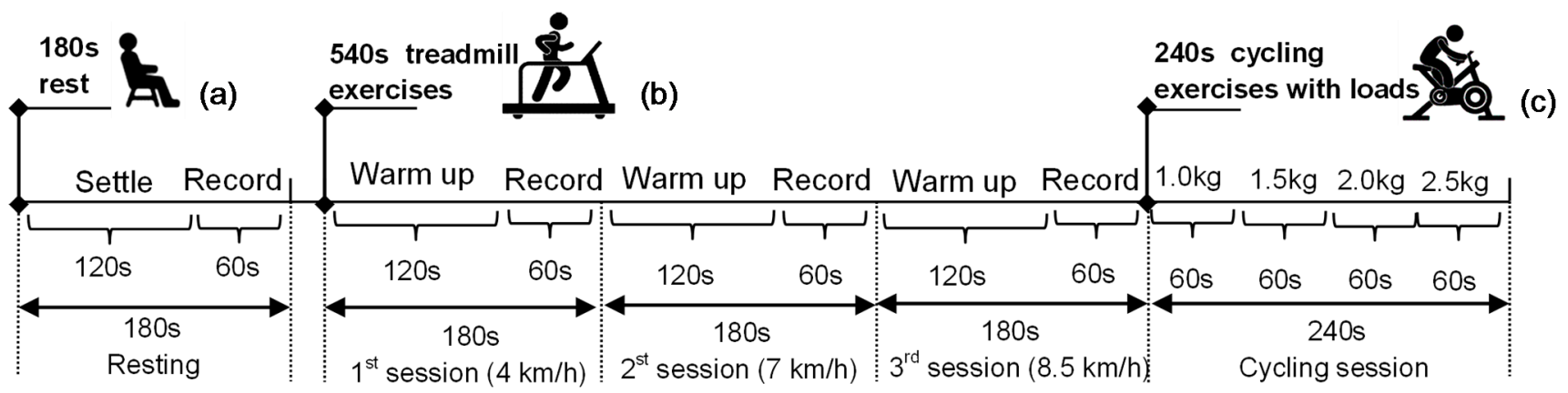

Figure 9. The illustrated protocol implementation of (a) resting on the chair with a period of $180 \mathrm{~s}$; (b) walking and running on treadmill with an execution period of $540 \mathrm{~s}$; and (c) cycling exercise with loads implemented in period of $240 \mathrm{~s}$.

\subsection{OEPS Measurement System}

The PPG signals were captured during the experimental measurements, and the system computed the HR every 15 second interval. All data sets from the OEPS were collected by the means of a 4-channel PPG board (DISCO4, Dialog Devices Ltd., Reading, Berkshire, UK). The ADC for these captured PPG signals was implemented by a 14bit data acquisition board (DAQ, USB-6009, National Instruments Co., Novato, CA, USA), and the control software of PPG board was performed by LabVIEW GUI (National Instruments Co., USA). The sampling frequency of $256 \mathrm{~Hz}$ was higher enough to reconstruct PPG signals within the Nyquist frequency condition, as well as the band pass filter of cut-off frequency $0.1-10 \mathrm{~Hz}$ were implemented to narrow down the region of interest of the signal. The desired signals were measured and PPG real time signals displayed on the screen of a PC as a waveform chart as shown in Figure 10.

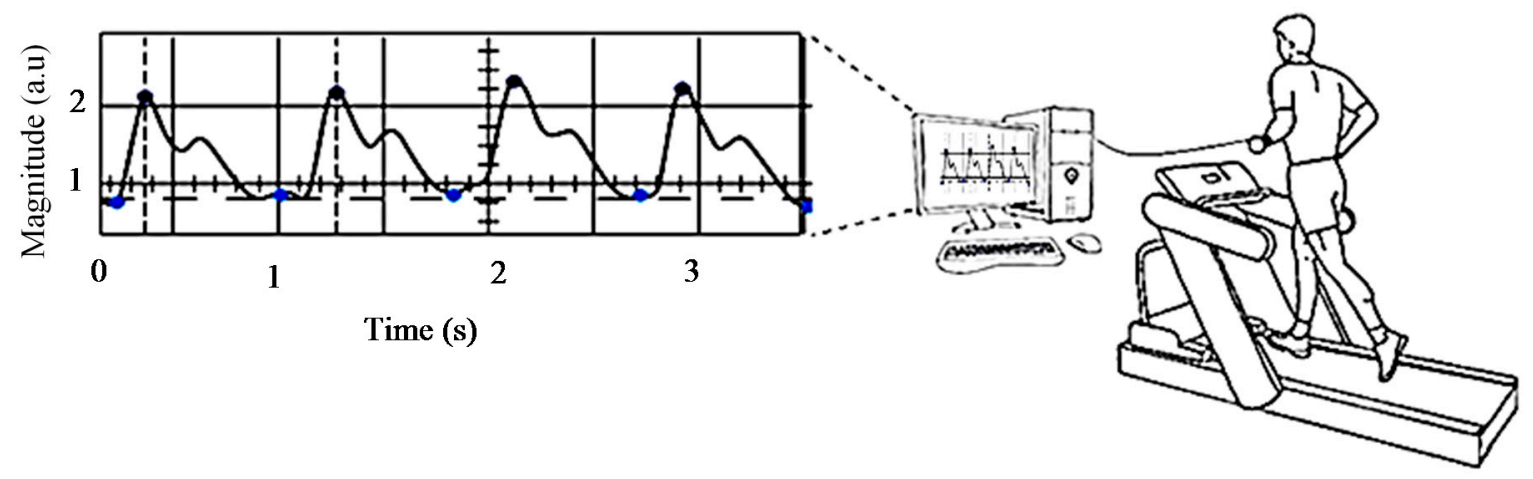

Figure 10. Physiological testing setup and platform, left is the LabView GUI and right is the treadmill.

\section{Results}

\subsection{Data Analysis of HR Detection}

Primary signal processing of the recorded datasets were executed using the Matlab software (The MathWorks, Inc., Natick, MA, USA) to evaluate the performance of the OEPS and commercial devices, for example, the Polar chest strap and Mio-Alpha wrist HR monitors. Two channel PPG 
signals from the OEPS and the acceleration reference were recorded by the execution program of LabVIEW. Figure 11 shows PPG signals captured from the palm attaching the OEPS with three wavelength illumination sources (i.e., green $525 \mathrm{~nm}$, red $650 \mathrm{~nm}$ and IR $870 \mathrm{~nm}$ ) during the stationary state where the participant was in the sitting position.

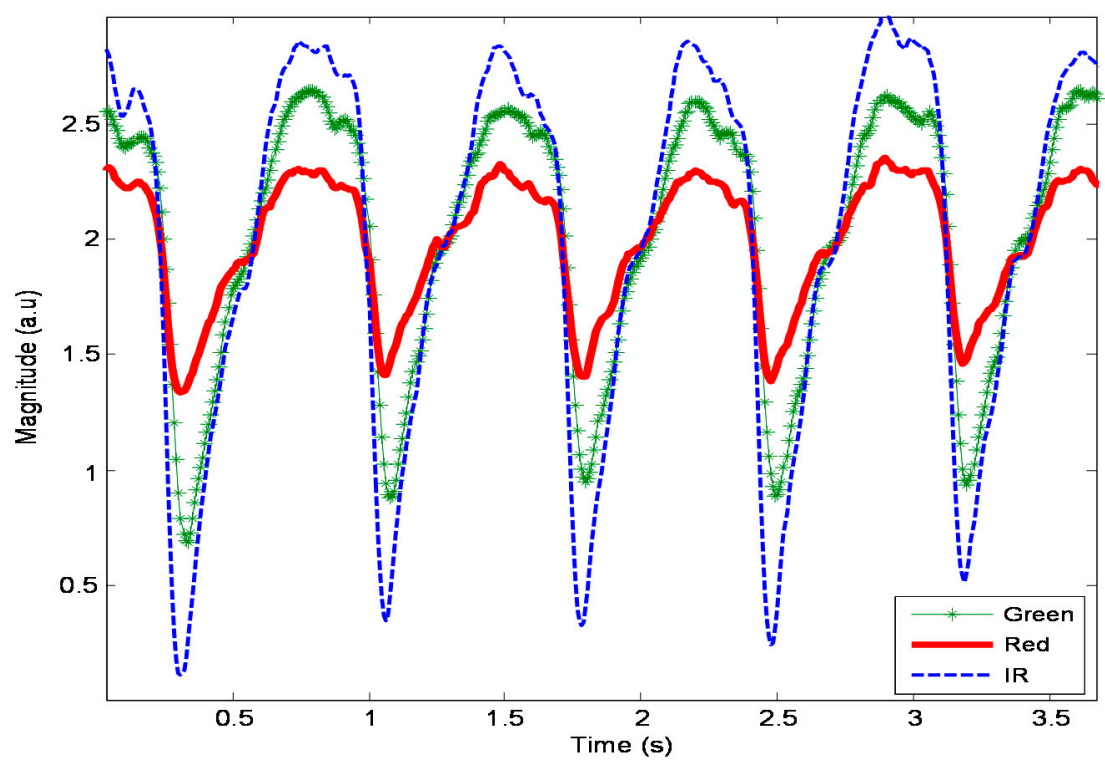

Figure 11. Photoplethysmography (PPG) signals captured by the OEPS with three wavelength illuminations.

HR can be extracted by an algorithm by separating the raw signals of three different wavelength illuminations and even perform pulse-pulse interval PPI from an individual wavelength illumination. The algorithm of PPG peak and trough-detection (APTRD) was developed with MatLab (The MathWorks, Inc., Natick, MA, USA) to extract pulsatile waveforms as PPG AC components resulting in the calculation of HR. Figure 12 shows the APTRD how to work out the trough detection from a single wavelength illumination. The algorithm runs to analyze each sample along with the duration period and to decide which samples are smallest than the nearest two neighbor samples. HR can be determined through the minimum point detected between two minimum troughs.

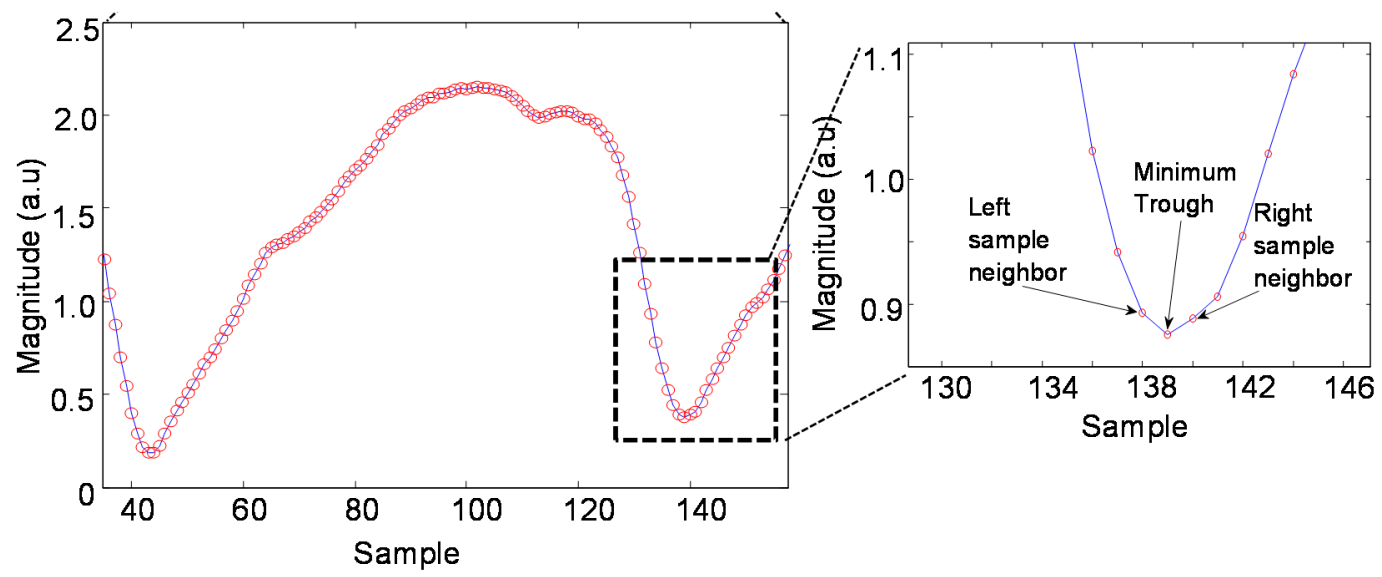

Figure 12. The algorithm of PPG peak and trough-detection (APTRD) developed to detect the trough of pulsatile waveform for HR calculation. 
The APTRD runs on the sample period and detects the minimum trough samples by setting three amplitude values and comparing them, previous value, current value and next value. Figure 13 shows a close look of different random sample intervals in waveforms. The results obtained from the preliminary processing procedure of trough detection values are well matched with the waveforms as presented in Figure 13a,b.

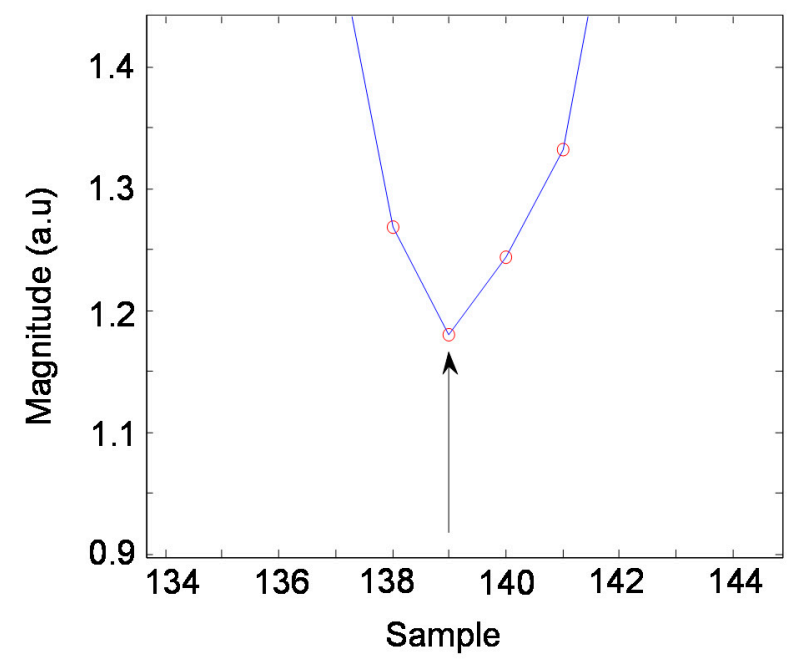

(a)

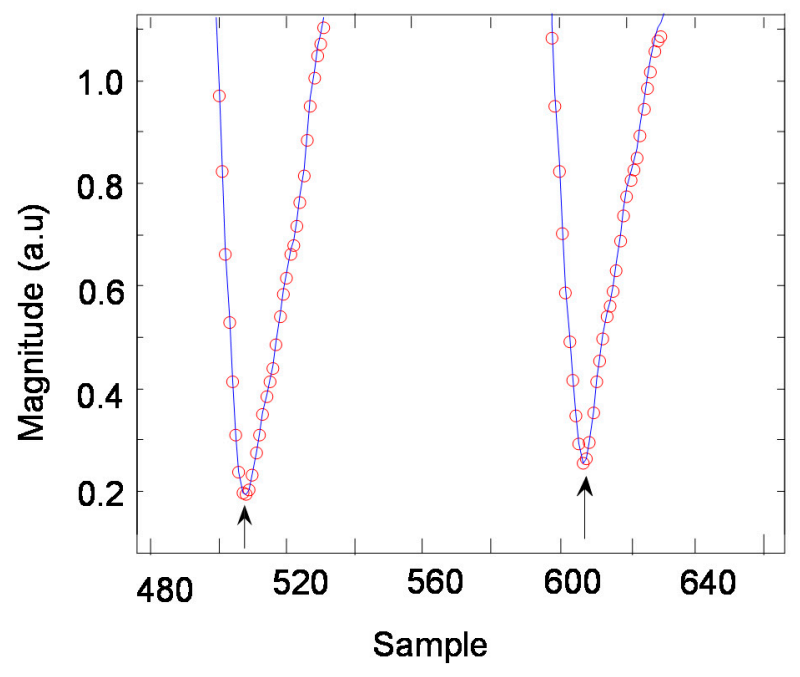

(b)

Figure 13. APTRD developed to detect a minimum valley of pulsatile features (a) at 139; (b) at 508 and at 607.

The resulting inter beats count derived from the APTRD and the average HR measurements were calculated over different epochs for the same pulsatile features of PPG signal as seen in Figure 14a,b.

Figure 14a indicates the beat account in duration samples of 600 samples is 6 beats and the HR is calculated as $76.8000 \approx 77$ beats per minute $(\mathrm{bpm})$. The algorithm was applied in different durations as displayed in Figure 14b and found that the beat account is 2 beats and the HR is $76.8000 \approx 77 \mathrm{bpm}$.

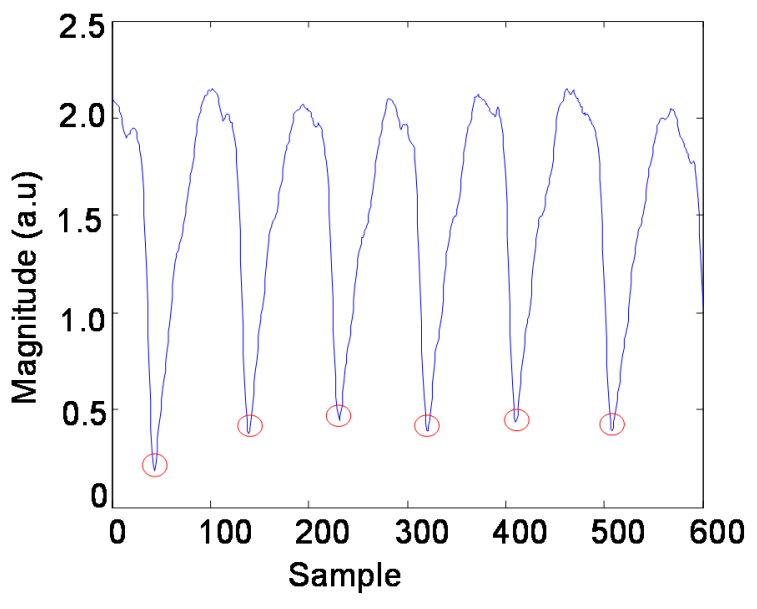

(a)

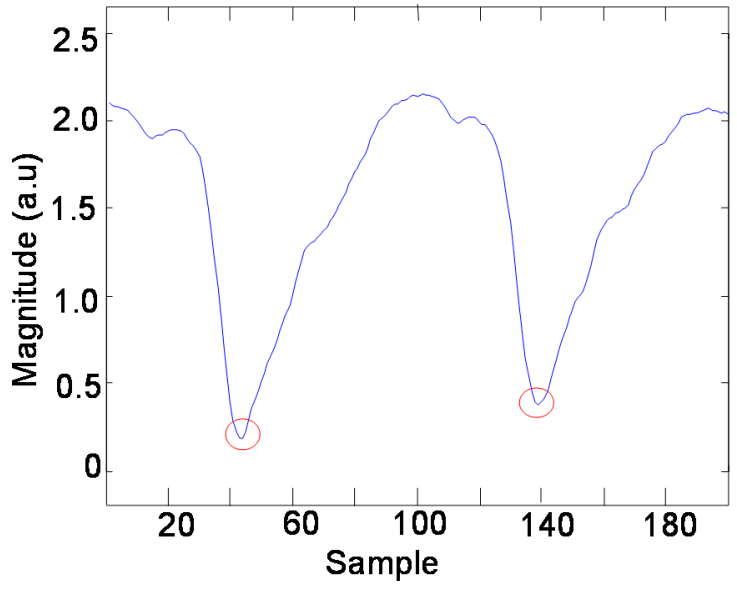

(b)

Figure 14. Inter beats count and average heart rate (HR) measurements from the pulsatile features of PPG signal. (a) Duration of 600 samples was detected as 6 beats count with $77 \mathrm{bpm}$; (b) Duration of 180 samples was counted 2 beats with $77 \mathrm{bpm}$. 
In the presence of movement, the PPG signal starts to get corrupted and as a result of this it becomes difficult to obtain physiological information. Motion artefact reduction (MAR) [32] was primarily introduced to recover the PPG signal and reduce motion artefact. Figure 15 shows the corrupted signal (Blue), recovered signal (Red) and reference 3MA signal (Black).

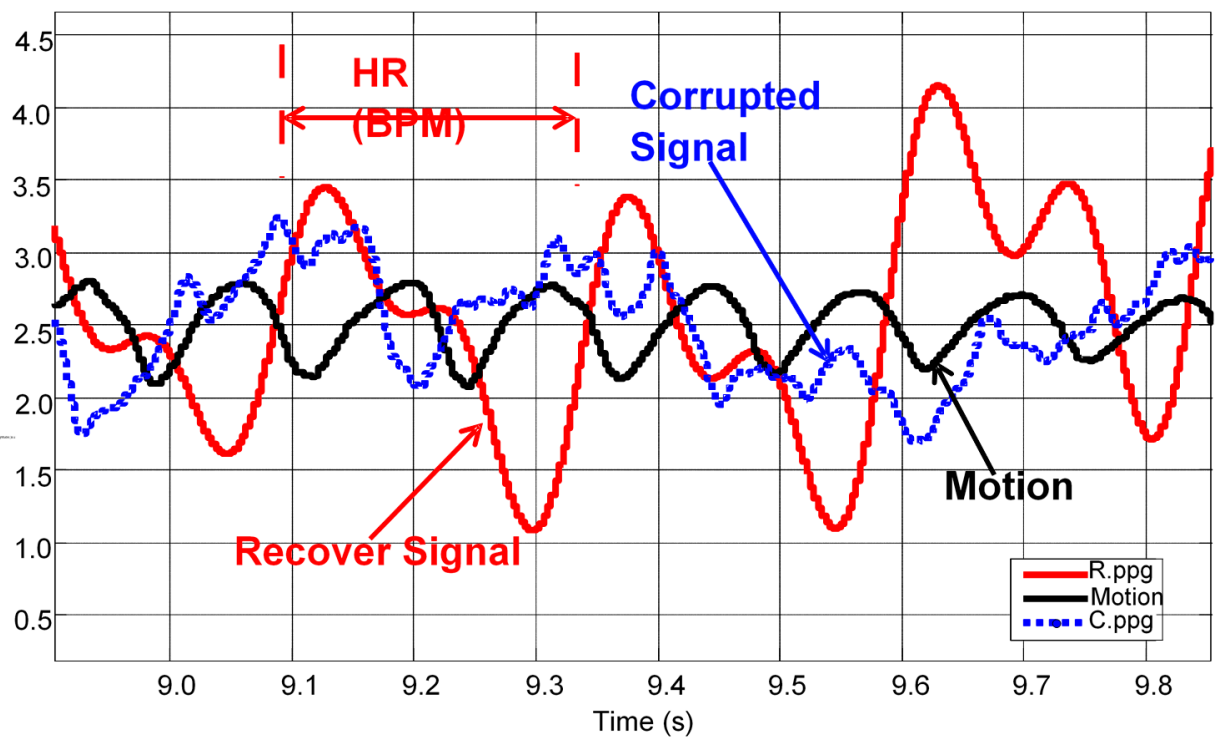

Figure 15. Recovery of PPG signals using motion artefact reduction (MAR).

Figure 16a shows PPG signals together with the reduced motion and Figure 16b synchronization with the criterion standard method of Polar electrocardiogram (ECG).

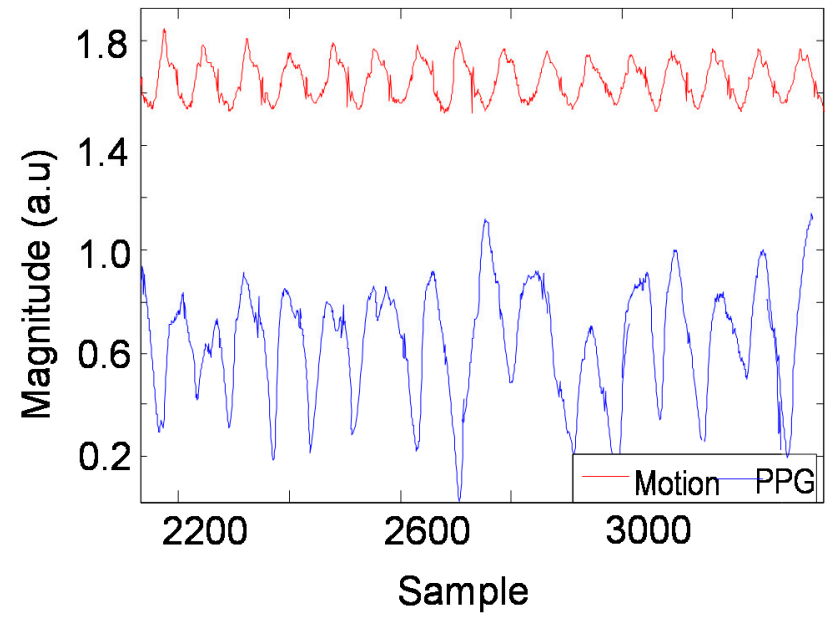

(a)

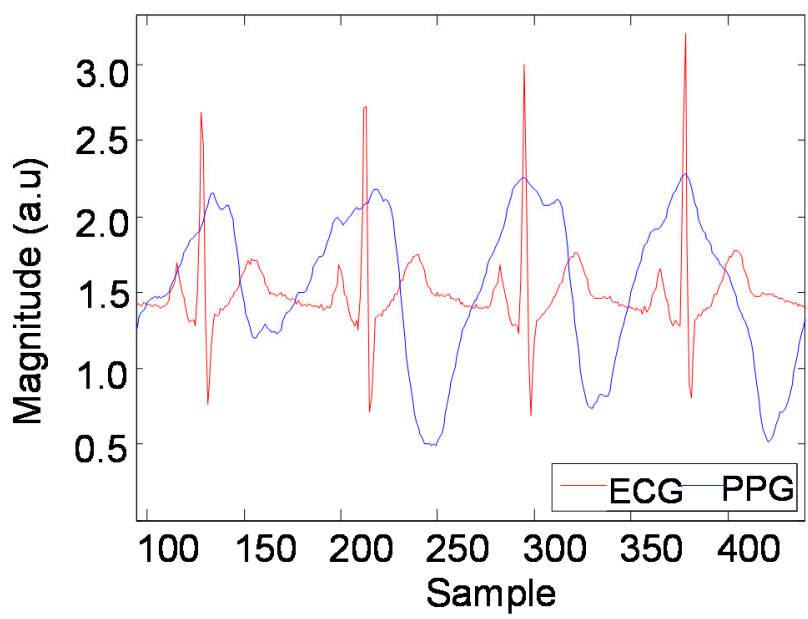

(b)

Figure 16. Recovery of PPG signals through motion artefact reduction (MAR) and synchronizing these PPG signals with Polar (ECG). (a) Recovering PPG with reference motion; (b) Synchronization between recovered PPG and golden standard ECG. 


\subsection{Statistical Analysis of HR between OEPS and Commercial Devices}

Statistical analysis of HR was performed using IBM SPSS Statistics 21 (SPSS Inc, Chicago, IL, USA). Statistical significance was accepted at $p<0.05$. All participant characteristics are presented as mean \pm standard deviation and standard error of the mean $(S E M)$. Student t-tests were performed between the HR data from Polar and the OEPS to determine when a difference was evident between the two devices. The same test was implemented between Mio-alpha and the OEPS in order to validate the data extracted from PPG signals of the OEPS. The relationships between Polar, Mio-alpha and the HR outputs from the OEPS were examined via correlation (r) analysis (Pearson product moment). The agreement between the two devices was evaluated by means of using Bland Altman's (BAA) 95\% limits of agreement approach. Table 1 summarizes a comparison study between Polar and the OEPS.

Table 1. Bias, 95\% limits of agreement (LOA) and correlation coefficient (r) of HR between Polar and the OEPS during Rest, Walking, Running and Cycling Activity, number of subjects $(n=16)$. Student paired t-tests for HR between Polar and the OEPS.

\begin{tabular}{|c|c|c|c|c|c|c|c|c|c|c|}
\hline & \multicolumn{2}{|c|}{ Polar } & \multicolumn{2}{|c|}{ OEPS } & \multirow{2}{*}{ Bias } & \multirow{2}{*}{$\mathbf{L O A}^{-}$} & \multirow{2}{*}{$\mathbf{L O A}^{+}$} & \multirow{2}{*}{$\mathbf{r}$} & \multirow{2}{*}{ Intercept } & \multirow{2}{*}{ Gradien } \\
\hline & Mean & SEM & Mean & SEM & & & & & & \\
\hline Rest & 72 & 3 & 71 & 3 & -1.13 & -6 & 3 & 0.99 & 6.50 & 0.92 \\
\hline Treadmill (movement) & 116 & 4 & 118 & 4 & 2.48 & -14 & 19 & 0.96 & 2.98 & 0.95 \\
\hline $4 \mathrm{~km} / \mathrm{h}^{\mathrm{a}}$ & 85 & 3 & 89 & 4 & 3.66 & -18 & 26 & 0.70 & 36.42 & 0.55 \\
\hline $7 \mathrm{~km} / \mathrm{h}$ & 117 & 3 & 118 & 3 & 1.56 & -10 & 13 & 0.89 & -4.55 & 1.03 \\
\hline $8.5 \mathrm{~km} / \mathrm{h}^{\mathrm{a}}$ & 144 & 4 & 148 & 4 & 2.33 & -13 & 17 & 0.89 & 23.84 & 0.82 \\
\hline Treadmill (still) & 119 & 5 & 118 & 5 & -1.08 & -23 & 20 & 0.93 & 6.74 & 0.95 \\
\hline $4 \mathrm{~km} / \mathrm{h}^{\mathrm{b}}$ & 88 & 3 & 89 & 4 & 1.15 & -14 & 17 & 0.84 & 33.51 & 0.61 \\
\hline $7 \mathrm{~km} / \mathrm{h}^{\mathrm{c}}$ & 121 & 5 & 119 & 4 & -2.83 & -13 & 8 & 0.94 & 5.16 & 0.98 \\
\hline $8.5 \mathrm{~km} / \mathrm{h}^{\mathrm{c}}$ & 147 & 6 & 148 & 6 & -1.75 & -35 & 31 & 0.62 & 57.77 & 0.62 \\
\hline Cycling & 135 & 3 & 133 & 3 & -3.11 & -21 & 15 & 0.93 & 5.64 & 0.98 \\
\hline $1 \mathrm{~kg}$ & 116 & 4 & 113 & 4 & -2.81 & -9 & 3 & 0.98 & 3.40 & 0.99 \\
\hline $1.5 \mathrm{~kg}$ & 129 & 5 & 126 & 5 & -2.38 & -12 & 7 & 0.97 & 10.33 & 0.94 \\
\hline $2 \mathrm{~kg}^{\mathrm{a}}$ & 144 & 6 & 141 & 5 & -2.53 & -16 & 11 & 0.96 & -15.86 & 1.13 \\
\hline $2.5 \mathrm{~kg}^{\mathrm{a}}$ & 158 & 6 & 153 & 5 & -4.80 & -38 & 28 & 0.69 & 44.59 & 0.74 \\
\hline
\end{tabular}

$\mathrm{LOA}^{-}$, Lower limits of agreement; $\mathrm{LOA}^{+}$, Upper limits of agreement. ${ }^{\mathrm{a}} \mathrm{n}=15 ;{ }^{\mathrm{b}} n=13 ;{ }^{\mathrm{c}} \mathrm{n}=12$.

Table 1 summarizes the mean values of HR presented by both Polar and the OEPS are comparable; a high linear association $(r \geq 0.96)$ was found between two measurements of HR as shown in Figure 17. No significant deference in HR was found between both devices $(p=0.73)$. The BAA plot of the difference (Polar-OEPS) against the mean value in HR is given by two methods ((Polar + OEPS)/2) is presented in Figure 18. The bias is B: $0.85 \mathrm{bpm}$, the standard deviation is SD: $9.20 \mathrm{bpm}$, and the limits of agreement (LOA) are in the range of $-17.18 \mathrm{bpm}$ to $+18.88 \mathrm{bpm}$ for lower and upper limits of agreement respectively. The $95 \%$ confidence intervals for the bias are $\mathrm{B} \pm 1.96 \mathrm{SD} \mathrm{bpm}$ for the lower $\left(\mathrm{LOA}^{-}\right)$, and the upper $\left(\mathrm{LOA}^{+}\right)$. All these intervals are reasonably narrow; as $95 \%$ of all (Polar-OEPS) data differences fall within the range of LOA. 


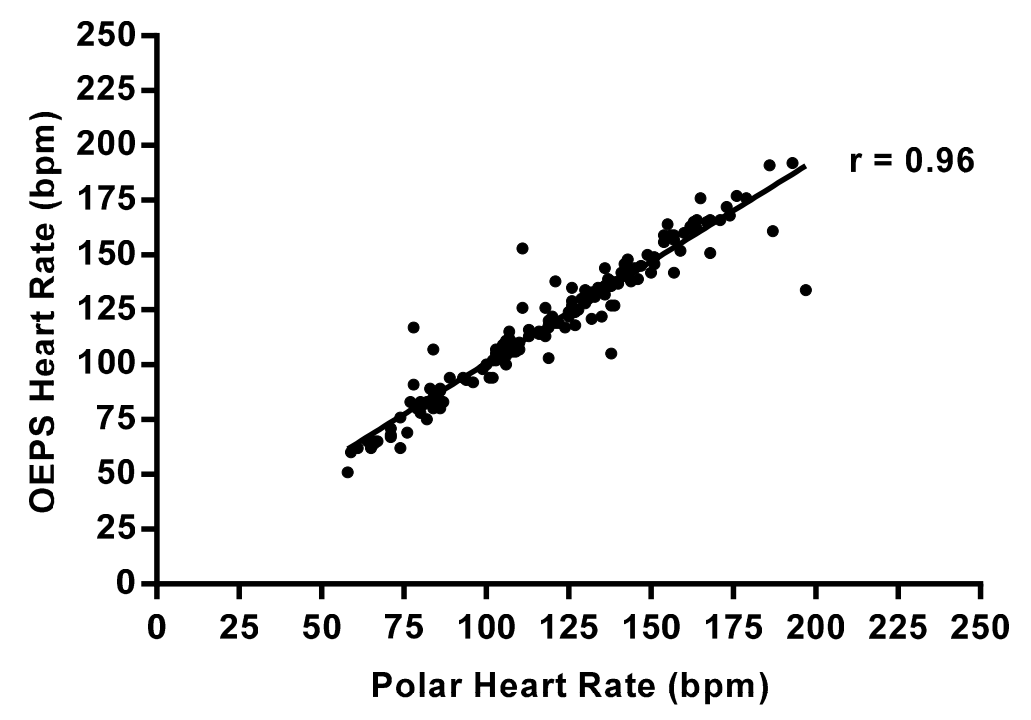

Figure 17. Correlation relationship between HR during rest and differing exercise modalities and intensities using Polar and the OEPS $(\mathrm{r}=0.96, p<0.0001)$.

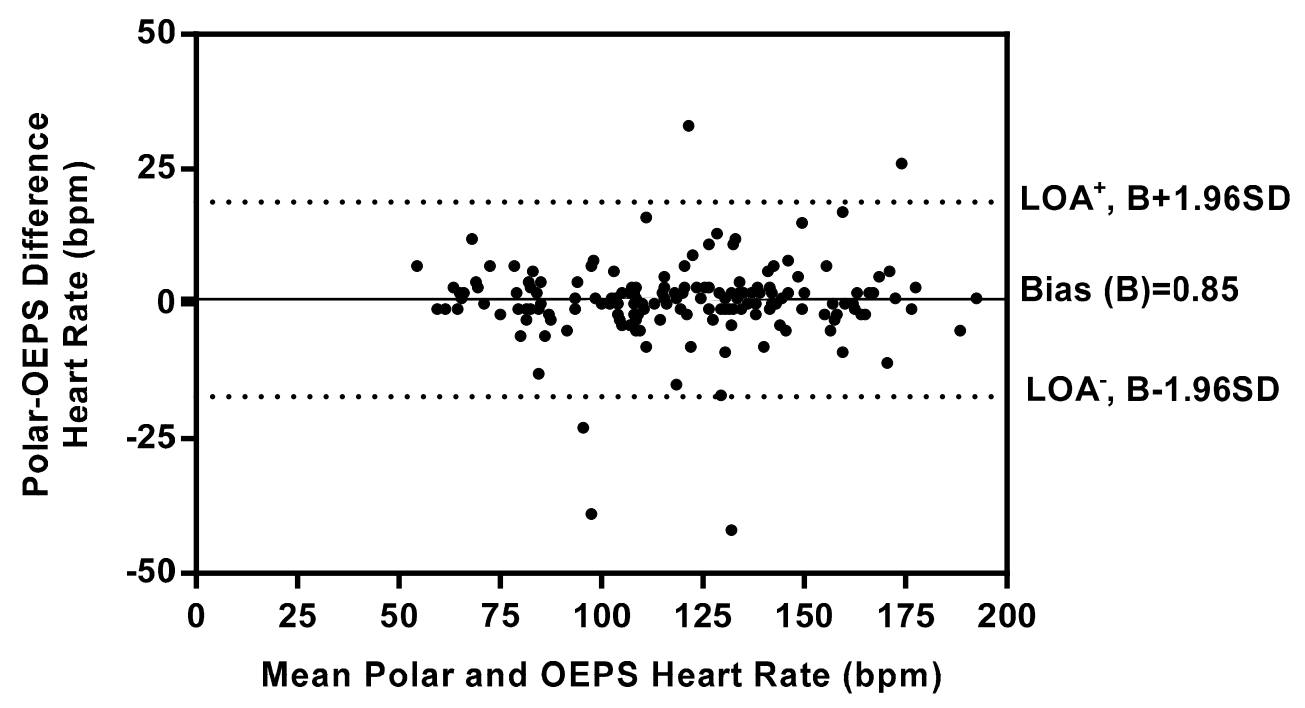

Figure 18. Bland-Altman plot shows differences in HR outputs recorded at rest and during differing exercise modalities and intensities using Polar and the OEPS. Mean bias (solid line) and limits of agreement (dashed line) are also shown.

The comparisons of HR between two measurement techniques, i.e., Mio-Alpha and the OEPS were performed using the t-test for paired data as shown in Table 2. Pearson's correlation analysis was also used to correlate quantitative variables $(\mathrm{r} \geq 0.96)$, as an indicator of two techniques evolving in parallel in Figure 19. The test shows there is no significant difference as probability value $(p=0.67)$. The Bland-Altman method was used to compare the values of HR obtained by the OEPS technique and the commercial HR monitoring using Mio-Alpha watch. Figure 20 shows the bias B: $1.63 \mathrm{bpm}$, standard deviation $\mathrm{SD}=8.62 \mathrm{bpm}$, lower and upper limits of agreement, $-15.27 \mathrm{bpm}$ and $+18.58 \mathrm{bpm}$ respectively. A histogram frequency distribution was used for all recorded data as shown in Figure 21. 
Table 2. Bias, 95\% limits of agreement (LOA) and correlation coefficient (r) of HR between Mio-Alpha and the OEPS during Rest, Walking, Running and Cycling Activity (16 subjects).

\begin{tabular}{|c|c|c|c|c|c|c|c|c|c|c|}
\hline & \multicolumn{2}{|c|}{ Mio-Alpha } & \multicolumn{2}{|c|}{ OEPS } & \multirow{2}{*}{ Bias } & \multirow{2}{*}{$\mathbf{L O A}^{-}$} & \multirow{2}{*}{$\mathbf{L O A}^{+}$} & \multirow{2}{*}{$\mathbf{r}$} & \multirow{2}{*}{ Intercept } & \multirow{2}{*}{ Gradient } \\
\hline & Mean & SEM & Mean & SEM & & & & & & \\
\hline Rest & 71 & 3 & 71 & 3 & 0.18 & -4 & 5 & 0.98 & -8.63 & 0.90 \\
\hline Treadmill (movement) & 119 & 4 & 118 & 4 & 2.48 & -14 & 19 & 0.96 & 6.98 & 0.92 \\
\hline $4 \mathrm{~km} / \mathrm{h}$ & 91 & 4 & 89 & 4 & 0.68 & -8 & 9 & 0.96 & -24.06 & 0.91 \\
\hline $7 \mathrm{~km} / \mathrm{h}$ & 116 & 3 & 118 & 3 & -2.37 & -19 & 15 & 0.74 & 8.42 & 0.30 \\
\hline $8.5 \mathrm{~km} / \mathrm{h}^{\mathrm{a}}$ & 150 & 4 & 148 & 4 & 2.33 & -13 & 17 & 0.89 & -23.93 & 0.59 \\
\hline Treadmill (still) & 120 & 5 & 118 & 5 & 1.39 & -23 & 20 & 0.93 & 9.74 & 0.94 \\
\hline $4 \mathrm{~km} / \mathrm{h}^{\mathrm{b}}$ & 89 & 3 & 89 & 4 & 0.42 & -13 & 14 & 0.89 & -1.77 & 0.51 \\
\hline $7 \mathrm{~km} / \mathrm{h}^{\mathrm{d}}$ & 122 & 5 & 119 & 4 & 3.25 & -10 & 17 & 0.92 & -9.12 & 0.55 \\
\hline $8.5 \mathrm{~km} / \mathrm{h}^{\mathrm{d}}$ & 150 & 6 & 148 & 6 & 4.91 & -21 & 31 & 0.76 & -33.84 & 0.29 \\
\hline Cycling & 135 & 3 & 133 & 3 & 2.47 & -21 & 15 & 0.93 & 4.64 & 0.98 \\
\hline $1 \mathrm{~kg}^{\mathrm{c}}$ & 113 & 3 & 113 & 4 & 1.00 & -9 & 11 & 0.95 & -7.20 & 0.68 \\
\hline $1.5 \mathrm{~kg}^{\mathrm{a}}$ & 128 & 5 & 126 & 5 & 2.86 & -7 & 13 & 0.96 & -14.00 & 0.77 \\
\hline $2 \mathrm{~kg}^{\mathrm{a}}$ & 142 & 5 & 141 & 5 & -1.21 & -5 & 8 & 0.98 & -2.02 & 0.80 \\
\hline $2.5 \mathrm{~kg}^{\mathrm{a}}$ & 157 & 6 & 153 & 5 & -4.66 & -29 & 39 & 0.63 & -22.56 & 0.17 \\
\hline
\end{tabular}

$\mathrm{LOA}^{-}$, Lower limits of agreement; LOA ${ }^{+}$, Upper limits of agreement. ${ }^{\mathrm{a}} n=15 ;{ }^{\mathrm{b}} n=14 ;{ }^{\mathrm{c}} n=13 ;{ }^{\mathrm{d}} n=12$.

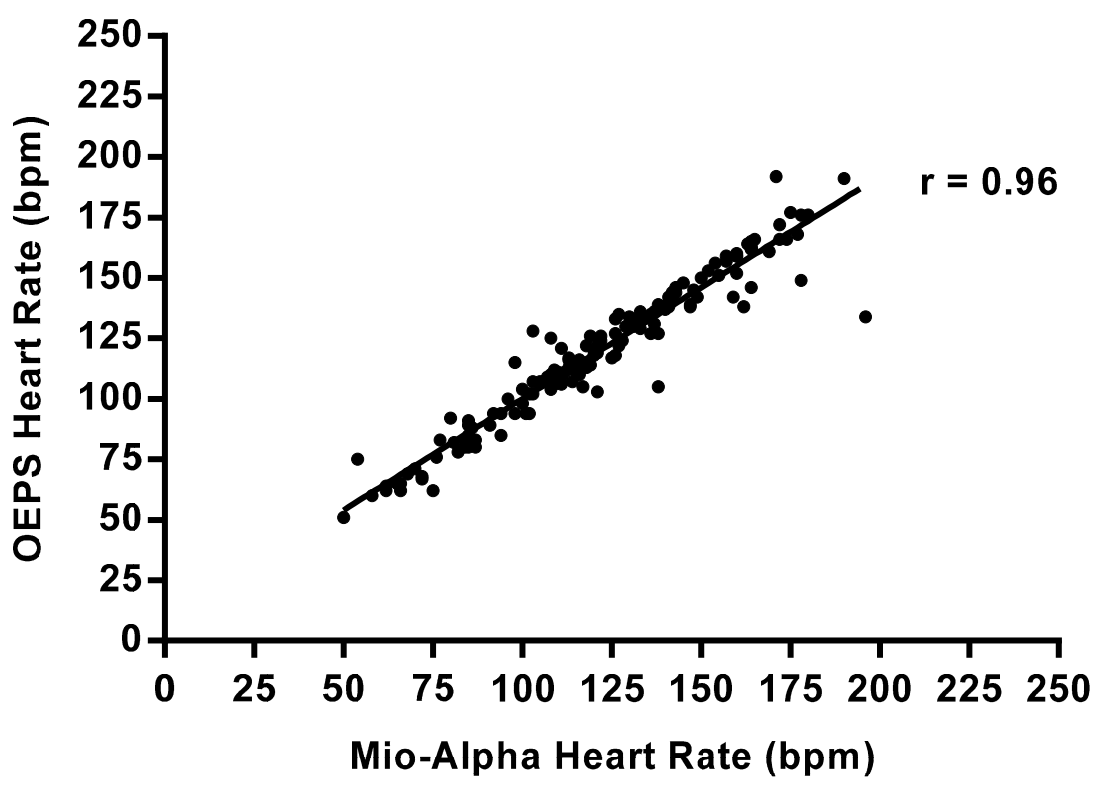

Figure 19. HR Correlation between Mio-Alpha and the OEPS $(\mathrm{r}=0.96, p<0.0001)$ during rest and differing exercise modalities and intensities. 


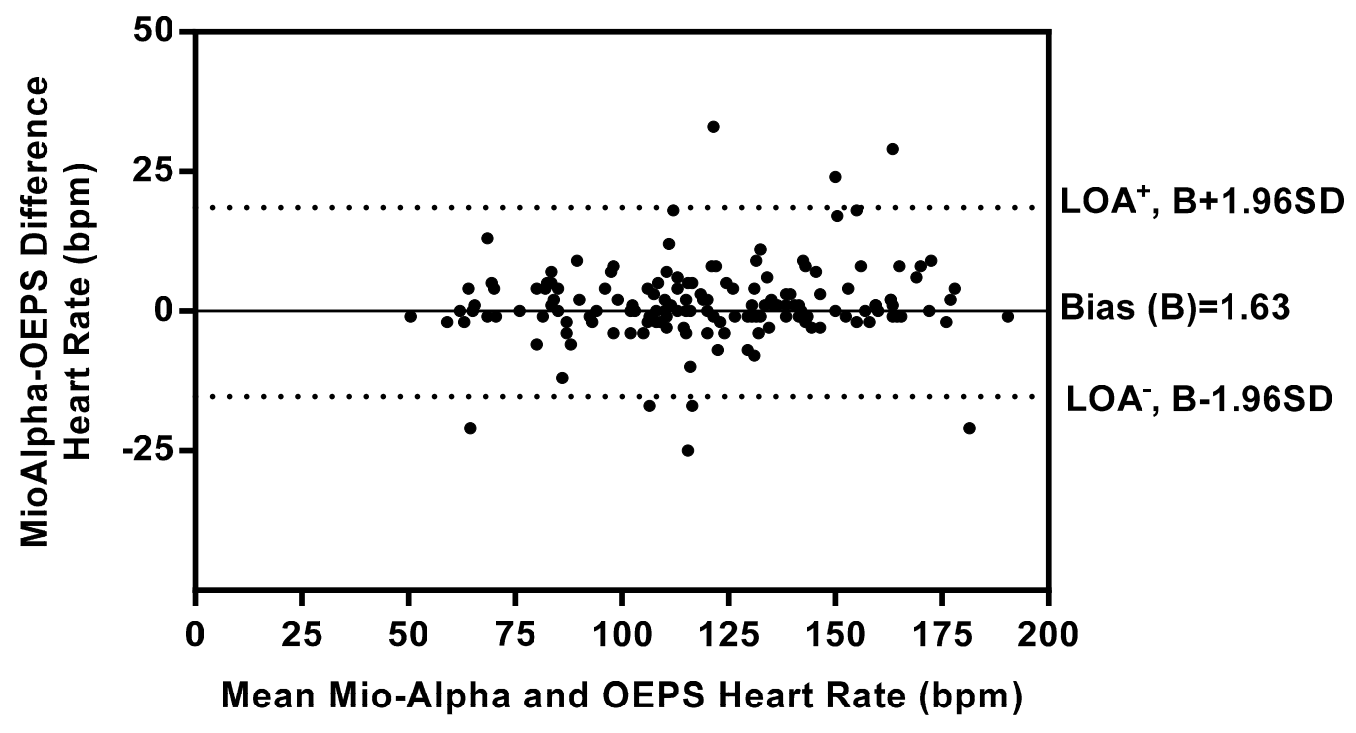

Figure 20. Bland-Altman plot shows differences in HR outputs recorded at rest and during differing exercise modalities and intensities using Mio-Alpha and the OEPS. Mean bias (solid line) and limits of agreement (dashed line).

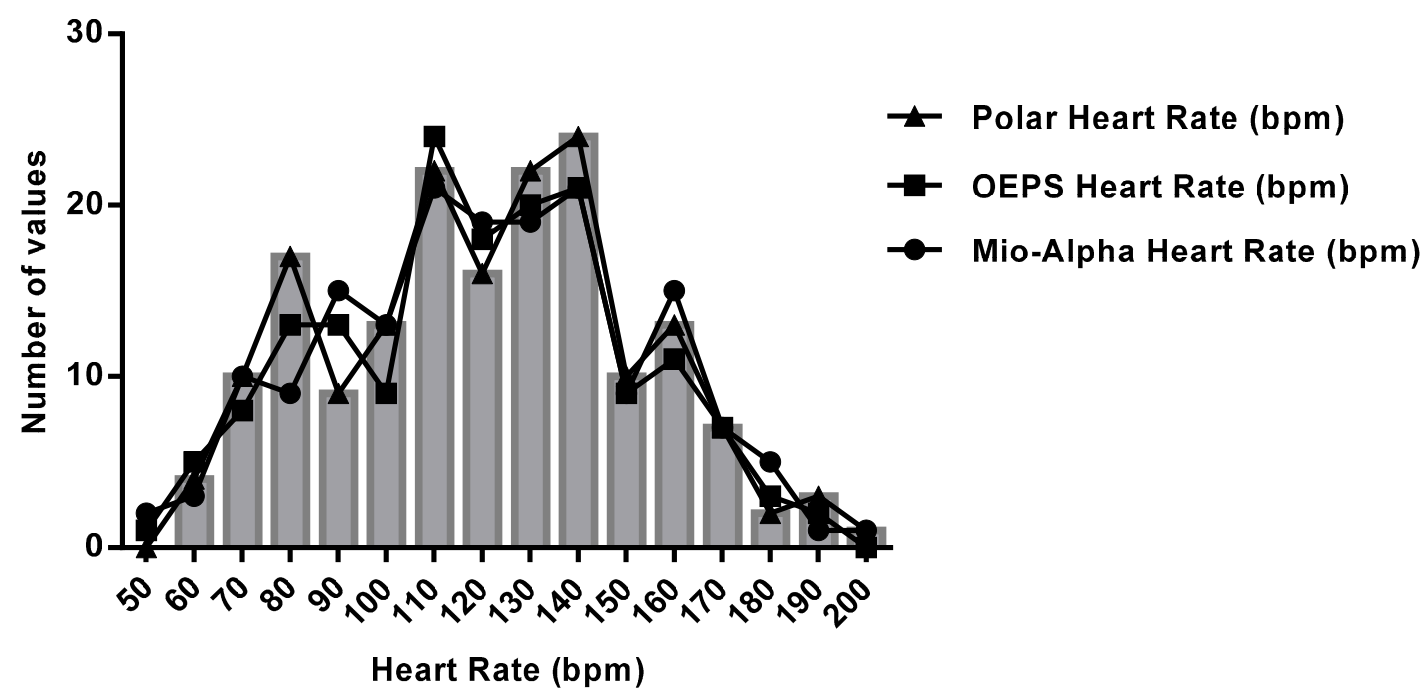

Figure 21. Histogram distribution for HR using Polar, Mio-Alpha and OEPS data.

\section{Discussion}

The algorithm for separating different wavelengths and detection of HR from PPG signals is efficient, despite the small SNR for both $650 \mathrm{~nm}$ and $870 \mathrm{~nm}$ wavelength illuminations during excessive movement. The APTRD for HR peak/trough detection works properly and this is evident because it calculates the HR accurately from the same waveform with different sample intervals. In addition, the APTRD provides valuable information such as the number of beats in a certain duration that could help in the determination of HRV to allow an assessment of cardiac nervous system by determining pulse to pulse interval (PPI) in a certain frequency domain. The results from the present study demonstrate the reliable PPG signals from the promising of the OEPS.

In this work, a six $532 \mathrm{~nm}$ LEDs was used to illuminate the designated tissue site due to its resistance against the corruption from a physical movement [42]. Moreover, the $532 \mathrm{~nm}$ wavelength 
LED with a narrower spectral band $( \pm 10 \mathrm{~nm})$ could be useful to effectively illuminate the peripheral blood vessels and to capture the pulsatile dynamic variation (PDV).

The comparisons of HR between measurement methods of (Polar and the OEPS) and (Mio-Alpha and OEPS) were performed using the t-test. The results show there is no significant difference between methods $(p>0.05)$. The correlation analysis demonstrates a strong relationship in HR measured using the Polar and the OEPS as well as Mio-Alpha and the OEPS (both $r=0.96$ and $p<0.0001)$.

The BAA method was applied to compare the values of HR obtained by the OEPS to the commercial devices, i.e., Polar and Mio-Alpha. The bias in HR measured using the OEPS and both the Polar and Mio-Alpha was found to be low and the limits of agreement are acceptable. The histogram of differences between the measurements of two methods presents a normal distribution. There is a slight difference among the data due to an invalid data set presented on the sensors (Polar, Mio-Alpha and the OEPS). The main reason for the distortion of the signal captured by the OEPS was inadequate attachment of the sensor; thus resulting in a misalignment of the area of interest.

\section{Conclusions}

This work introduced an OEPS together with an opto-electronic system and an algorithm, i.e., APTRD/MAR for accurate detection of HR and eliminating noise signals caused by motion artefacts respectively. Both the OEPS and the APTRD/MAR indeed enhance the quality of PPG signal to determine HR during route activities or an exercise. The APTRD converts the PPG signals collected during a cycling and treadmill running exercise into a HR value. The commercial devices (Polar and Mio-Alpha) were used to validate the OEPS derived HR. The good agreement analysis shows the comparability of the both techniques of the OEPS and commercial methods in the evaluation of HR. The limitation of invalid data or signal error that appears due to misalignment could be overcome by the means of a proper packaging for the OEPS. The outcome from the proposed OEPS indicates a new paradigm of opto-physiological monitoring technique as ideally suited to be incorporated into wearable devices.

\section{Acknowledgments}

The authors acknowledge and would like to thank EPSRC Shaping Capability Funds - Bridging the Gaps projects 2012, and Wolfson School, Loughborough University as well as The Ministry of Higher Education in the Kingdom of Saudi Arabia represented at Taif University for this study. This research was also supported by the National Institute for Health Research (NIHR) Diet, Lifestyle \& Physical Activity Biomedical Research Unit based at University Hospitals of Leicester and Loughborough University.

\section{Author Contributions}

Abdullah Alzahrani designed experimental protocol and implemented the experiments, carried out the signal processing and wrote the paper. Sijung Hu supervised the project and guided the project implementation, and organised paper writing. Vicente Azorin-Peris established the opto-electronic 
hardware, supported the signal processing and proofread the paper. Dale Esliger provided the measurement and analytical expertise for the comparator accelerometer and heart rate sensors. Laura Barrett organized the healthy subjects to participate in the study. All authors contributed extensively to the work presented in this paper.

\section{Conflicts of Interest}

The authors declare no conflict of interest.

\section{References}

1. Bonato, P. Clinical applications of wearable technology. IEEE Proc. Eng. Med. Biol. 2009, 3, 6580-6583.

2. Teichmann, D.; Matteis, D.; Bartelt, T.; Walter, M.; Leonhardt, S. A Bendable and Wearable Cardiorespiratory Monitoring Device Fusing Two Noncontact Sensor Principles. IEEE J. Biomed. Health Inform. Heal. Inf. 2015, 19, 784-793.

3. Perk, J.; Backer, G.D.; Gohlke, H. European Guidelines on cardiovascular disease prevention in clinical practice. Eur. Heart J. 2012, 33, 1635-1701.

4. Teichmann, D.; Kuhn, A.; Leonhardt, S.; Walter, M. The MAIN Shirt: A textile-integrated magnetic induction sensor array. Sensors 2014, 14, 1039-1056.

5. Venema, B.; Blazek, V.; Blanik, N.; Schiefer, J.; Leonhardt, S. Evaluating innovative in-ear pulse oximetry for unobtrusive cardiovascular and pulmonary monitoring during sleep. IEEE J. Trans. Eng. Heal. Med. 2013, 1, 2168-2372.

6. Achten, J.; Jeukendrup, E. Heart rate monitoring, applications and limitations. Sports Med. 2003, $33,517-538$.

7. Keytel, R.; Goedecke, H.; Noakes, D.; Hiiloskorpi, H.; Laukkanen, R.; Merwe, L.; Lambert, V. Prediction of energy expenditure from heart rate monitoring during submaximal exercise. J. Sport Sci. 2005, 23, 289-297.

8. Venema, B.; Gehring, H.; Michelsen, I.; Blanik, N.; Blazek, V.; Leonhardt, S. Robustness, Specificity, and Reliability of an In-Ear Pulse Oximetric Sensor in Surgical Patients. IEEE J. Biomed. Health Inform. Heal. Inf. 2014, 18, 1178-1185.

9. Hu, S.; Azorin-Peris, V.; Zheng J. Opto-physiological modeling applied to photoplethysmographic cardiovascular assessment. J. Healthc. Eng. 2009, 4, 505-528.

10. Hu, S.; Sun, Y.; Azorin-Peris, V.; Greenwald, S.; Chambers, J.; Zhu, Y. Motion-compensated noncontact imaging photoplethysmography to monitor cardiorespiratory status during exercise. J. Biomed. Opt. 2011, 16, 1-9.

11. Anliker, U.; Ward, A.; Lukowicz, P.; Tröster G.; Dolveck, F.; Baer, M.; Keita, F.; Schenker, B.; Catarsi, F.; Coluccini, L.; et al. AMON: A wearable multiparameter medical monitoring and alert system. IEEE Trans. Inf. Technol. Biomed. 2004, 8, 415-427.

12. Nonin Medical Inc. NoninWristOx 3100. Medical Product Service GmbH Nonin; Nonin Medical Inc.: Plymouth, MN, USA, 2006.

13. Fruin, L.; Rankin, W. Validity of a multi-sensor armband in estimating rest and exercise energy expenditure. Med. Sci. Sports Exerc. 2004, 36, 1063-1069. 
14. Park, S.; Jayaraman, S. E-Health and quality of life: The role of the Wearable Motherboard. Stud. Health Technol. Inform. 2004, 108, 239-252.

15. Grossman, P. The Life Shirt: A multi-function ambulatory system monitoring health, disease, and medical intervention in the real world. Stud. Health Technol. Inform. 2004, 108, 133-141.

16. Pandian, S.; Mohanavelu, K.; Safeer P.; Kotresh, M.; Shakunthala, T.; Parvati, G.; Padaki, C. Smart Vest: Wearable multi-parameter remote physiological monitoring system. Med. Eng. Phys. 2008, 30, 466-477.

17. Lukowicz, P.; Anliker, U.; Ward, J.; Troster, G. AMON: A Wearable Medical Computer for High Risk Patients. In Proceedings of the 6th International Symposium on Wearable Computers (ISWC 02), Seattle, WA, USA, 7-10 October 2002; Volume 8, pp. 7695-1816.

18. Lee, W.; Jung, G.; Lee, K. The periodic moving average filter for removing motion artifacts from PPG signals. Int. J. Control Autom. Syst. 2007, 6, 701-706.

19. Tamura, T.; Maeda, Y; Sekine, M; Yoshida, M. Wearable Photoplethysmographic Sensors_-Past and Present. J. Electron. 2014, 3, 282-302.

20. Yoo, K.; Kim, S. Motion artifact reduction in photoplethysmography using independent component analysis. IEEE Trans. Biomed. Eng. 2006, 53, 566-568.

21. Natarajan, B.; Krishnan, R.; Warren, S.; Two-stage approach for detection and reduction of motion artifacts in photoplethysmographic data. IEEE Trans. Biomed. Eng. 2010, 57, 1867-1876.

22. Yao, J.; Warren, S. A short study to assess the potential of independent component analysis for motion artifact separation in wearable pulse oximeter signal. In Proceedings of the 27th Annual International Conference of the Engineering in Medicine and Biology Society (EMBS), Shanghai, China, 1-4 September 2005; pp. 3585-3588.

23. Stoica, P.; Moses, L. Spectral Analysis of Signals; Prentice Hall: Upper Saddle River, NJ, USA, 2005.

24. Ram, M.; Madhav, V.; Krishna, H.; Komalla, R.; Reddy, A. A novel approach for motion artifact reduction in PPG signals based on AS-LMS adaptive filter. IEEE Trans. Instr. Meas. 2012, 61, 1445-1457.

25. Yousefi, R.; Nourani, M.; Ostadabbas, S.; Panahi, I. A motion-tolerant adaptive algorithm for wearable photoplethysmographic biosensors. J. Biomed. Health Inform. 2014, 18, 670-681.

26. Fukushima, H.; Kawanaka, H.; Bhuiyan, S.; Oguri, K. Estimating heart rate using wrist-type photoplethysmography and acceleration sensor while running. In Proceedings of the Annual International Conference of the IEEE Engineering in Medicine and Biology Society (EMBC), San Diego, CA, USA, 28 August-1 September 2012; pp. 2901-2904.

27. Lee, B.; Han, J.; Baek, J.; Shin, Park, S.; Yi, J. Improved elimination of motion artifacts from a photoplethysmographic signal using a kalman smoother with simultaneous accelerometry. Physiol. Meas. 2010, 31, 1585-1603.

28. Sun, X.; Yang, P.; Li, Y.; Gao, Z; Zhang, Y. Robust heart beat detection from photoplethysmography interlaced with motion artifacts based on empirical mode decomposition. In Proceedings of the IEEE-EMBS International Conference on Biomedical and Health Informatics (BHI), Hong Kong, China, 5-7 January 2012; pp. 775-778.

29. Hayes, J.; Smith, R. A new method for pulse oximetry possessing inherent insensitivity to artifact. IEEE Trans. Biol. Med. Eng. 2001, 48, 452-461. 
30. Raghuram, M.; Madhavi; V.; Krishna, H.; Redd, A. Evaluation of wavelets for reduction of motion artifacts in photoplethysmographic signals. IEEE Inf. Sci. 2010, 2, 460-463.

31. Yan, Y.; Poon, C.; Zhang, Y. Reduction of motion artifact in pulse oximetry by smoothed pseudo wigner-ville distribution. J. NeuroEng. Rehabil. 2005, 2, 1186-1743.

32. Peng, F.; Zhang, Z.; Gou, X.; Liu, H.; Wang, W. Motion artifact removal from photoplethysmographic signals by combining temporally constrained independent component analysis and adaptive filter. Biomed. Eng. 2014, 13, 1186-1475.

33. Reddy, A.; George, B.; Kumar, J. Use of fourier series analysis for motion artifact reduction and data compression of photoplethysmographic signals. IEEE Trans. Instrum. Meas. 2009, 58, $1706-1711$.

34. Poh, Z.; Swenson, C.; Picard, W. Motion-tolerant magnetic earring sensor and wireless earpiece for wearable photoplethysmography. IEEE Trans. Inf. Technol. Biomed. 2010, 14, 786-794.

35. Lopez, M.; Giannetti, R.; Dotor, L.; Silveira, P.; Golmayo, D.; Miguel, F.; Bilbao, A.; Canales, M.; Pilar, M. Heuristic algorithm for photoplethysmographic heart rate tracking during maximal exercise test. J. Med. Biol. Eng. 2012, 32, 181-188.

36. Hu, S.; Azorin-Peris, V. Opto-Physiological Sensor and Method of Design. GB Patent Application GB2, 519,335, 22 April 2014.

37. Hu, S.; Azorin Peris, V.; Zheng, J. A study of opto-physiological modelling to quantify tissue absorbance in imaging photoplethysmography. In Proceedings of the Annual International Conference of the IEEE Engineering in Medicine and Biology Society (EMBC), Buenos Aires, Argentina, 31 August-4 September 2010; pp. 5776-5779.

38. Alzahrani, A; Hu, S.; Azorin-Peris, V. A Comparative Study of Physiological Monitoring with a Wearable Opto-Electronic Patch Sensor (OEPS) for Motion Reduction. J. Biosens. 2015, 5, 288-307.

39. Hu, S.; Shi, P.; Azorin-Peris, V.; Echiadis, A.; Zheng, J.; Zhu, Y.; Cheang, Y. Non-Contact Reflection Photoplethysmography towards Effective Human Physiological Monitoring. J. Med. Biol. Eng. 2010, 30, 161-167.

40. Hu, S.; Azorin Peris, V.; Echiadis, A.; Zheng, J.; Shi, P. Development of effective photoplethysmographic measurement techniques: From contact to non-contact and from point to imaging. In Proceedings of the Annual International Conference of the IEEE Engineering in Medicine and Biology Society, Minneapolis, MN, USA, 3-6 September 2009; pp. 6550-6553.

41. Belkin, M.; Schwartz, M. New Biological Phenomena Associated with Laser Radiation. Health Phys. 1989, 56, 687-690.

42. Lee, J.; Matsumura, K.; Yamakoshi, K.; Rolfe, P.; Tanaka, S.; Yamakoshi, T. Comparison between Red, Green and Blue Light Reflection Photoplethysmography for Heart Rate Monitoring during Motion. In Proceedings of the 35th Annual International Conference of the IEEE Engineering in Medicine and Biology Society (EMBC), Osaka, Japan, 3-7 July 2013; pp. 1724-1727.

(C) 2015 by the authors; licensee MDPI, Basel, Switzerland. This article is an open access article distributed under the terms and conditions of the Creative Commons Attribution license (http://creativecommons.org/licenses/by/4.0/). 